\title{
An algorithm for the elastic flow of surfaces
}

\author{
RALUCA E. RUSU ${ }^{\dagger}$ \\ Institut für Angewandte Mathematik, Universität Freiburg, \\ Hermann-Herder-Strasse 10, 79104 Freiburg, Germany
}

[Received 10 December 2003 and in revised form 22 February 2005]

\begin{abstract}
A semi-implicit fully discrete finite element scheme for the computation of the parametric elastic flow of two-dimensional surfaces in $\mathbb{R}^{3}$ based on a variational form is presented. Linear finite elements are used for the space discretization of a mixed formulation. Time discretization is carried out by a semi-implicit method to linearize the problem.
\end{abstract}

\section{Introduction}

The elastic flow is the evolution of a surface which is determined by the steepest descent of the $L^{2}$-gradient of the classical bending energy

$$
E(\Gamma)=\frac{1}{2} \int_{\Gamma} H^{2} \mathrm{~d} o
$$

where $H$ denotes the sum of the principal curvatures of the surface $\Gamma$. This formula comes from elasticity theory. In the physical sense the problem can be interpreted as the motion of an elastic body, idealised to a surface, which possesses bending energy and moves to minimize its energy. The model does not take into account the fact that the moving surface also has kinetic energy. The energy $E$ is also known as the Willmore functional after the name of the mathematician T. J. Willmore who was among the first to study it from the mathematical point of view (see [14, 15, 16]).

Starting with the energy functional $E$ an abstract initial value problem can be formulated: for a given smooth, regular, closed initial surface $\Gamma_{0}$ find a family $(\Gamma(t))_{t \geqslant 0}$ of surfaces $\Gamma(t), t \geqslant 0$, which describes the evolution of $\Gamma_{0}$ under the $L^{2}$-gradient $E^{\prime}$ of the energy functional and satisfies $\Gamma(0)=\Gamma_{0}$. This means that each point of the surface $\Gamma(t), t \geqslant 0$, moves with the time and space dependent velocity $v$ which is determined by $E^{\prime}$ in the following way: if we choose a parametrization $X(\cdot, t): S \rightarrow \Gamma(t)$ of the surface $\Gamma(t)$ where $S$ is a suitable reference surface, then

$$
(v, \varphi)_{L^{2}(\Gamma(t))}=-\left.\frac{\mathrm{d}}{\mathrm{d} \epsilon} E((X(\cdot, t)+\epsilon \varphi)(S))\right|_{\epsilon=0}=-\left\langle E^{\prime}(\Gamma(t)), \varphi\right\rangle
$$

for suitable test functions $\varphi: S \rightarrow \mathbb{R}^{3}$ where $(\cdot, \cdot)_{L^{2}(\Gamma(t))}$ is the $L^{2}(\Gamma(t))$-scalar product. The first variation of $E$ is denoted by $\left\langle E^{\prime}(\Gamma(t)), \varphi\right\rangle$ and delivered by the directional gradient of the functional. A time dependent, nonlinear problem of fourth order in space arises.

The velocity condition (1) leads in the case of a two-dimensional moving surface to

$$
v \cdot v+\Delta_{\Gamma} H+H\left(\frac{1}{2} H^{2}-2 K\right)=0
$$

\footnotetext{
$\dagger$ The author was supported by the German Science Foundation (DFG) via Graduiertenkolleg Nichtlineare Differentialgleichungen: Modellierung, Theorie, Numerik, Visualisierung. E-mail: raluca.rusu@mathematik.uni-freiburg.de
} 
on $\Gamma$ (see [16]), which is the law of motion for the normal velocity $v \cdot v$ where $v$ is the inner unit normal, $\Delta_{\Gamma}$ is the Laplace-Beltrami or the surface Laplacian operator and $K$ is the Gaussian curvature of $\Gamma$.

For the elastic flow of curves in $\mathbb{R}^{n}, n \geqslant 2$, global existence in time of a solution was proved (see [11] for $n=2$ and [7] for $n \geqslant 3$ ). Existence of a solution for the evolution of two-dimensional surfaces in $\mathbb{R}^{n}, n \geqslant 3$, was shown in [10] up to a time $T<\infty$, where $T$ depends on the curvatures of the initial surface $\Gamma_{0}$. Singularities arising in the evolution of two-dimensional surfaces were treated in [13]. There it is verified numerically that singularities can appear in finite time.

According to (1) the problem has variational form. Assuming enough differentiability it is easy to see that the energy equality

$$
\int_{\Gamma(t)}|v|^{2} \mathrm{~d} o+\frac{1}{2} \frac{\mathrm{d}}{\mathrm{d} t} \int_{\Gamma(t)} H^{2}(t) \mathrm{d} o=0
$$

holds since the velocity $v$ is $X_{t}(t)$. Thus, (1) is suitable for the use of finite element methods. Finite element solutions for the elastic flow of curves in plane and space have been implemented in [7]. A mixed formulation is used there for the position vector and the curvature vector. This is important for the use of piecewise linear finite elements.

We shall focus in this article on the motion of two-dimensional, oriented, not necessarily embedded, surfaces in $\mathbb{R}^{3}$ which can be of different topological types. We shall assume that the surfaces can be parametrized over a suitable surface $S$. A numerical method for the computation of the elastic flow of surfaces is described and obtained as follows. We first introduce the necessary differential geometry. In Section 2 the velocity condition (1) is determined for the parametric elastic flow. To do that, expressions which contain merely geometrical quantities are calculated for the first variation of the energy functional. In doing so, we seek a way to handle the Gaussian curvature $K$. We look for a mixed formulation which is suitable for discretization with piecewise linear finite elements as in [7]. Consequently, we succeed in eliminating $K$ and obtain instead the tangential gradient of the normal $v$ (see Lemma 2.1). Since the tangential gradient of $v$ is still not sufficient for discretization with piecewise linear finite elements, we shift this derivative to the other terms by partial integration. The reflection in the surface will thus appear in the formula for the first variation of $E$ (see Lemma 2.2) and therefore in the velocity condition. We formulate a mixed problem where the free variables are the position vector of the surface and the mean curvature vector (see Problem 2.3). This is suitable for discretization with piecewise linear finite elements. The resulting velocity is normal on $\Gamma(t)$ and fulfils (2). The mixed problem is valid for the elastic flow of curves as well.

In Section 3 a semi-discrete and a fully discrete method are built based on the variational form of the mixed problem (see Problems 3.1] and 3.2). These are derived analogously to [7] by using piecewise linear finite elements for the space discretization and a semi-implicit method for the time discretization. Thus, a system of linear equations has to be solved at each time step.

Suitable finite element methods for evolutionary parametric surfaces are described in [6] and [12]. The evolution by mean curvature has been treated there. A discretization via finite elements for the Laplace-Beltrami operator on arbitrary surfaces can be found in [5]. A boundary value problem with smooth boundary conditions is defined in [3] as an extension of the result of this article. It is applied to surface restoration. Numerical simulations are also presented. In [4] the authors prove error estimates for a time continuous finite element method for the Willmore flow in the graph representation. A mixed formulation is used there for the evolution of two-dimensional graphs having as variables the height and a weighted mean curvature. Test calculations are presented. 
Here is a list of some important technical remarks. Consider a two-dimensional, compact $C^{k, \chi}$ surface $\Gamma \subset \mathbb{R}^{3}$ for $k \in \mathbb{N}, 0<\chi<1$ and $\chi=1$ if $k=0$. We find a finite $C^{k, \chi}$ atlas of open subsets $U_{i} \subset \Gamma$ such that $\Gamma=\bigcup_{i=1}^{i_{0}} U_{i}$ (see [17, p. 64]). For each $U_{i}$ there exists a homeomorphic $C^{k, \chi}$ map $X_{i}: \Omega_{i} \rightarrow U_{i}$ of an open subset $\Omega_{i} \subset \mathbb{R}^{2}$ onto $U_{i}, i=1, \ldots, i_{0}$. Since $X_{i}$ is a homeomorphism and $C^{k, \chi}$, a unit normal vector $v$ exists at each point of the surface $\Gamma$ for $k \geqslant 1$ and almost everywhere if $\Gamma$ is Lipschitz. We say in this case that the surface is regular. In the case of a compact surface with a continuous unit normal field (see [2] p. 86]), we assume that $v$ is the inner unit normal vector. The map $X_{i}: \Omega_{i} \rightarrow \Gamma$ is called a local parametrization of $\Gamma$. We define a two-dimensional regular compact $C^{k}, k \geqslant 1$, surface $\Gamma \subset \mathbb{R}^{3}$ analogously. If $\varphi: \Gamma \rightarrow \mathbb{R}$ is a smooth function, then by definition $\varphi \circ X_{i}: \Omega_{i} \rightarrow \mathbb{R}$ is smooth for all $i=1, \ldots, i_{0}$.

We define the tangential gradient of $\varphi$ with respect to $\Gamma$ at a point of $\Gamma$ with normal $v$ as

$$
\nabla_{\Gamma} \varphi:=D \varphi-D \varphi \cdot v v
$$

where $D \varphi$ is the usual gradient of $\varphi$ (see [9, p. 389]). For this definition $\varphi$ is assumed to be differentiable in an open neighbourhood of $\Gamma \subset \mathbb{R}^{3}$. Nevertheless the tangential gradient depends only on the values of $\varphi$ on $\Gamma$. Furthermore the Laplace-Beltrami operator of $\varphi$ on a $C^{2}$ surface $\Gamma$ is the tangential divergence of the tangential gradient of $\varphi$, i.e.

$$
\Delta_{\Gamma} \varphi:=\nabla_{\Gamma} \cdot \nabla_{\Gamma} \varphi
$$

Here we have used the differentiability of an extension of the normal $v$ of $\Gamma$ over an open neighbourhood of $\Gamma \subset \mathbb{R}^{3}$ (see [9, p. 355]). One can define these geometrical quantities by means of a local parametrization of $\Gamma$ as well (see [1, p. 4]). Thus, if $X: \Omega \rightarrow \Gamma$ is a local parametrization of a smooth surface $\Gamma$, then

$$
\nabla_{\Gamma} \varphi=\sum_{i, j=1}^{2}\left(g^{i j}(\varphi \circ X)_{u_{i}} X_{u_{j}}\right) \circ X^{-1}
$$

and

$$
\Delta_{\Gamma} \varphi=\left(\frac{1}{\sqrt{\operatorname{det} g}} \sum_{i, j=1}^{2}\left(\sqrt{\operatorname{det} g} g^{i j}(\varphi \circ X)_{u_{i}}\right)_{u_{j}}\right) \circ X^{-1}
$$

pointwise on $X(\Omega)$ for a smooth function $\varphi: \Gamma \rightarrow \mathbb{R}$. Here $g=\left(g_{i j}\right)_{i, j=1,2}, \sqrt{\operatorname{det} g}$ and $\left(g^{i j}\right)_{i, j=1,2}=g^{-1}$ are the first fundamental form, the surface element and the inverse of the first fundamental form of $\Gamma$ in the parametric representation respectively (see [2, p. 76]).

We denote by $H$ the mean curvature and by $K$ the Gaussian curvature of a regular $C^{2}$ surface $\Gamma$. We define $H$, respectively $K$, to be the sum, respectively the product, of the principal curvatures of $\Gamma$ (see [2, p. 115]). Thus, it follows that

$$
H=\Delta_{\Gamma} X \cdot v=-\nabla_{\Gamma} X \cdot \nabla_{\Gamma} v=-v \cdot \frac{v_{u} \times X_{v}+X_{u} \times v_{v}}{\left|X_{u} \times X_{v}\right|}
$$

for a local parametrization $X: \Omega \rightarrow \Gamma$ of $\Gamma$ mapping $(u, v) \in \Omega$ to $X(u, v) \in \Gamma$. We remark that $a \times b \in \mathbb{R}^{3}$ is the vector product of $a, b \in \mathbb{R}^{3}$. Furthermore

$$
K=v \cdot \frac{v_{u} \times v_{v}}{\left|X_{u} \times X_{v}\right|}
$$


and

$$
H v=\Delta_{\Gamma} X=-\frac{v_{u} \times X_{v}+X_{u} \times v_{v}}{\left|X_{u} \times X_{v}\right|}
$$

pointwise in $X(\Omega)$ where $H v$ is the mean curvature vector of $\Gamma$.

We note that, if $\varphi: \Gamma \rightarrow \mathbb{R}^{3}$ is a smooth vector-valued function, then $\nabla_{\Gamma} \varphi$ is the matrix

$$
\nabla_{\Gamma} \varphi=\left(\nabla_{j} \varphi_{i}\right)_{i, j=1,2,3} \in \mathbb{R}^{3 \times 3}
$$

with the rows $\nabla_{\Gamma} \varphi_{i}, i=1,2,3$, where $\nabla_{j} \varphi_{i}$ is the $j$ th component of the tangential gradient of $\varphi_{i}$, $i, j=1,2,3$, and $\Delta_{\Gamma} \varphi$ is the column vector $\left(\Delta_{\Gamma} \varphi_{1}, \Delta_{\Gamma} \varphi_{2}, \Delta_{\Gamma} \varphi_{3}\right)^{t} \in \mathbb{R}^{3}$. We also point out that we distinguish between the left and right product of a matrix with a vector. Thus, if $v \in \mathbb{R}^{3}$ is a vector and $A \in \mathbb{R}^{3 \times 3}$ a matrix, then $(v A)_{j}=v_{i} a_{i j}, j=1,2,3$. Moreover

$$
\nabla_{\Gamma} \varphi \cdot \nabla_{\Gamma} \psi=\nabla_{i} \varphi_{j} \nabla_{i} \psi_{j}
$$

and

$$
\Delta_{\Gamma} \varphi \cdot \Delta_{\Gamma} \psi=\Delta_{\Gamma} \varphi_{i} \Delta_{\Gamma} \psi_{i}
$$

where the summation convention is used and $\varphi, \psi: \Gamma \rightarrow \mathbb{R}^{3}$ are smooth.

We mention furthermore the geometric relations

$$
\Delta_{\Gamma} v=-\left|\nabla_{\Gamma} v\right|^{2} v-\nabla_{\Gamma} H
$$

and

$$
\left|\nabla_{\Gamma} v\right|^{2}=H^{2}-2 K
$$

which are valid pointwise in $\Gamma$.

If $\varphi: \Gamma \rightarrow \mathbb{R}$ is a function such that $\varphi \circ X$ is integrable in $\Omega$ for a local parametrization $X: \Omega \rightarrow \Gamma$ of a regular Lipschitz surface $\Gamma$, then we define the surface integral over $X(\Omega)$ as

$$
\int_{\Gamma \cap X(\Omega)} \varphi \mathrm{d} o:=\int_{\Omega} \varphi \circ X\left|X_{u} \times X_{v}\right| \mathrm{d} u \mathrm{~d} v
$$

where the integral on the right is the usual Lebesgue integral. The formula for partial integration on $\Gamma$ is

$$
\int_{\Gamma} \nabla_{\Gamma} \varphi \mathrm{d} o=-\int_{\Gamma} \varphi H v \mathrm{~d} o,
$$

which can be proved by using 44,211 , providing the closed surface $\Gamma$ is $C^{2}$. By using the product rule and (12) we obtain

$$
\int_{\Gamma} \Delta_{\Gamma} \varphi \psi \mathrm{d} o=-\int_{\Gamma} \nabla_{\Gamma} \varphi \cdot \nabla_{\Gamma} \psi \mathrm{d} o
$$

where $\psi: \Gamma \rightarrow \mathbb{R}$ is another smooth and integrable function. We will identify in the following a function $\varphi$ on $X(\Omega) \subset \Gamma$ with $\varphi \circ X$ on $\Omega$.

We apply the usual notation $L^{2}(\Gamma)^{3}$ and $H^{l}(\Gamma)^{3}$ for a regular $C^{k, \chi}$ surface $\Gamma, l \in \mathbb{N}, l \leqslant k+\chi$, which are defined as in [17, p. 92]. This means that we still have the space $H^{1}(\Gamma)^{3}$ in the case of a Lipschitz surface $\Gamma$.

There are different topological types of surfaces. It is convenient to parametrize globally the surface depending on its topological type. For example, if the type is 0 , then $X: S^{2} \rightarrow \Gamma$ will be a global parametrization where $S^{2}$ is the unit sphere in $\mathbb{R}^{3}$. 


\section{Continuous formulation}

We are interested in the evolution of surfaces under the gradient flow of the bending energy functional. Thus, to explain the velocity condition defined in (1), the first variation of the functional has to be calculated.

Let $\Gamma$ be a regular $C^{3}$ surface with normal $\nu$. For simplicity we assume that

$$
X: \Omega \rightarrow \Gamma \subset \mathbb{R}^{3}
$$

is a global parametrization over an open domain $\Omega \subset \mathbb{R}^{2}$. Because of $(8)$ we write

$$
E(X)=\frac{1}{2} \int_{\Gamma}\left|\Delta_{\Gamma} X\right|^{2} \mathrm{~d} o
$$

for the energy functional $E$ of $\Gamma=X(\Omega)$. We obtain the following expression for the first variation of $E$.

LEMmA 2.1 Let $X: \Omega \rightarrow \Gamma \subset \mathbb{R}^{3}$ be a parametrization of the regular $C^{3}$ surface $\Gamma$ such that $\Gamma=X(\Omega)$ and let $E$ be the energy functional defined in $(13)$. Then

$$
\begin{aligned}
\left\langle E^{\prime}(X), \varphi\right\rangle & =\left.\frac{\mathrm{d}}{\mathrm{d} \epsilon} E(X+\epsilon \varphi)\right|_{\epsilon=0} \\
& =\int_{\Gamma} \Delta_{\Gamma} X \cdot\left(\Delta_{\Gamma} \varphi+2 v \nabla_{\Gamma} v \cdot \nabla_{\Gamma} \varphi\right) \mathrm{d} o+\frac{1}{2} \int_{\Gamma}\left|\Delta_{\Gamma} X\right|^{2} \nabla_{\Gamma} X \cdot \nabla_{\Gamma} \varphi \mathrm{d} o
\end{aligned}
$$

for all $\varphi \in C_{0}^{2}(\Omega)^{3}$.

Proof. Let $\varphi \in C_{0}^{2}(\Omega)^{3}$ be an arbitrary function and consider the surfaces $\Gamma_{\epsilon}:=X_{\epsilon}(\Omega):=$ $(X+\epsilon \varphi)(\Omega)$ for arbitrarily small $\epsilon \in \mathbb{R}$. We need to calculate

$$
\left.\frac{\mathrm{d}}{\mathrm{d} \epsilon} E\left(X_{\epsilon}\right)\right|_{\epsilon=0}=\left.\frac{1}{2} \frac{\mathrm{d}}{\mathrm{d} \epsilon} \int_{\Gamma_{\epsilon}}\left|\Delta_{\Gamma_{\epsilon}} X_{\epsilon}\right|^{2} \mathrm{~d} o\right|_{\epsilon=0} .
$$

Using (11) and the product rule gives

$$
\begin{aligned}
\left.\frac{\mathrm{d}}{\mathrm{d} \epsilon} E\left(X_{\epsilon}\right)\right|_{\epsilon=0}= & \left.\frac{1}{2} \frac{\mathrm{d}}{\mathrm{d} \epsilon} \int_{\Omega}\left|\Delta_{\Gamma_{\epsilon}} X_{\epsilon}\right|^{2}\left|X_{\epsilon u} \times X_{\epsilon v}\right| \mathrm{d} u \mathrm{~d} v\right|_{\epsilon=0} \\
= & \left.\frac{1}{2} \int_{\Omega} \frac{\mathrm{d}}{\mathrm{d} \epsilon}\left|\Delta_{\Gamma_{\epsilon}} X_{\epsilon}\right|^{2}\right|_{\epsilon=0}\left|X_{u} \times X_{v}\right| \mathrm{d} u \mathrm{~d} v \\
& +\left.\frac{1}{2} \int_{\Omega}\left|\Delta_{\Gamma} X\right|^{2} \frac{\mathrm{d}}{\mathrm{d} \epsilon}\left|X_{\epsilon u} \times X_{\epsilon v}\right|\right|_{\epsilon=0} \mathrm{~d} u \mathrm{~d} v .
\end{aligned}
$$

By (8) we have

$$
\left.\frac{1}{2} \frac{\mathrm{d}}{\mathrm{d} \epsilon}\left|\Delta_{\Gamma_{\epsilon}} X_{\epsilon}\right|^{2}\right|_{\epsilon=0}=\left.\frac{1}{2} \frac{\mathrm{d}}{\mathrm{d} \epsilon}\left|\frac{v_{\epsilon u} \times X_{\epsilon v}+X_{\epsilon u} \times v_{\epsilon v}}{\left|X_{\epsilon u} \times X_{\epsilon v}\right|}\right|^{2}\right|_{\epsilon=0}
$$

where

$$
v_{\epsilon}=\frac{X_{\epsilon u} \times X_{\epsilon v}}{\left|X_{\epsilon u} \times X_{\epsilon v}\right|},
$$


pointwise in $\Omega$, is the unit normal vector of $\Gamma_{\epsilon}$. Due to the quotient rule and

$$
\left.\frac{\mathrm{d}}{\mathrm{d} \epsilon} v_{\epsilon}\right|_{\epsilon=0}=\frac{\varphi_{u} \times X_{v}+X_{u} \times \varphi_{v}}{\left|X_{u} \times X_{v}\right|}-v \nabla_{\Gamma} X \cdot \nabla_{\Gamma} \varphi
$$

we get

$$
\begin{aligned}
\left.\frac{1}{2} \frac{\mathrm{d}}{\mathrm{d} \epsilon}\left|\Delta_{\Gamma_{\epsilon}} X_{\epsilon}\right|^{2}\right|_{\epsilon=0} & \\
= & -\Delta_{\Gamma} X \cdot \frac{1}{\left|X_{u} \times X_{v}\right|}\left(\left(\frac{\varphi_{u} \times X_{v}+X_{u} \times \varphi_{v}}{\left|X_{u} \times X_{v}\right|}\right)_{u} \times X_{v}+X_{u} \times\left(\frac{\varphi_{u} \times X_{v}+X_{u} \times \varphi_{v}}{\left|X_{u} \times X_{v}\right|}\right)_{v}\right) \\
& -\Delta_{\Gamma} X \cdot \frac{v_{u} \times \varphi_{v}+\varphi_{u} \times v_{v}}{\left|X_{u} \times X_{v}\right|}-2\left|\Delta_{\Gamma} X\right|^{2} \nabla_{\Gamma} X \cdot \nabla_{\Gamma} \varphi
\end{aligned}
$$

pointwise in $\Omega$. Inserting the above into 15 leads to

$$
\begin{aligned}
\frac{\mathrm{d}}{\mathrm{d} \epsilon} E\left(X_{\epsilon}\right) & \left.\right|_{\epsilon=0} \\
= & -\int_{\Omega} \Delta_{\Gamma} X \cdot\left(\left(\frac{\varphi_{u} \times X_{v}+X_{u} \times \varphi_{v}}{\left|X_{u} \times X_{v}\right|}\right)_{u} \times X_{v}+X_{u} \times\left(\frac{\varphi_{u} \times X_{v}+X_{u} \times \varphi_{v}}{\left|X_{u} \times X_{v}\right|}\right)_{v}\right) \mathrm{d} u \mathrm{~d} v \\
& -\int_{\Omega} \Delta_{\Gamma} X \cdot\left(v_{u} \times \varphi_{v}+\varphi_{u} \times v_{v}\right) \mathrm{d} u \mathrm{~d} v-\frac{3}{2} \int_{\Omega}\left|\Delta_{\Gamma} X\right|^{2} \nabla_{\Gamma} X \cdot \nabla_{\Gamma} \varphi \mathrm{d} o .
\end{aligned}
$$

We expect from (2) that a derivative of fourth order of a parametrization appears in the first variation of $E$. Due to (5) we obtain

$$
\begin{aligned}
\Delta_{\Gamma} \varphi= & \left\{\frac { 1 } { | X _ { u } \times X _ { v } | } \left(-\left(\frac{\left(\varphi_{u} \times X_{v}+X_{u} \times \varphi_{v}\right) \times X_{v}}{\left|X_{u} \times X_{v}\right|}\right)_{u}-\left(\frac{X_{u} \times\left(\varphi_{u} \times X_{v}+X_{u} \times \varphi_{v}\right)}{\left|X_{u} \times X_{v}\right|}\right)_{v}\right.\right. \\
& \left.\left.+\left(v \times \varphi_{v}\right)_{u}+\left(\varphi_{u} \times v\right)_{v}+\left(\frac{\varphi_{u} \cdot X_{v}-\varphi_{v} \cdot X_{u}}{\left|X_{u} \times X_{v}\right|}\right)_{u} X_{v}+\left(\frac{-\varphi_{u} \cdot X_{v}+\varphi_{v} \cdot X_{u}}{\left|X_{u} \times X_{v}\right|}\right)_{v} X_{u}\right)\right\}
\end{aligned}
$$

pointwise in $\Omega$. By inserting the Laplace-Beltrami $\Delta_{\Gamma} \varphi$ of $\varphi$ into the first variation of $E$ and by using the $C^{3}$ differentiability of $\Gamma$ we make the term containing $\nabla_{\Gamma} v$ appear in $(14)$. It follows that

$$
\left.\frac{\mathrm{d}}{\mathrm{d} \epsilon} E\left(X_{\epsilon}\right)\right|_{\epsilon=0}=\int_{\Gamma} \Delta_{\Gamma} X \cdot\left(\Delta_{\Gamma} \varphi+2 \nu \nabla_{\Gamma} v \cdot \nabla_{\Gamma} \varphi\right) \mathrm{d} o+\frac{1}{2} \int_{\Gamma}\left|\Delta_{\Gamma} X\right|^{2} \nabla_{\Gamma} X \cdot \nabla_{\Gamma} \varphi \mathrm{d} o,
$$

which is the claim of the lemma.

For practical purposes (see next section), it is important to remark that in Lemma 2.1 the test function $\varphi$ is arbitrary, i.e. $\varphi$ is allowed to have tangential components.

For the sake of completeness we note that, if we assume $\varphi$ to be normal on $\Gamma$ throughout the proof of Lemma 2.1. use (7) and shift the derivatives of $\varphi$ to the other terms, in case $\Gamma$ is $C^{4}$, we obtain (2) for the normal velocity.

Let us return to the elastic flow. The goal is to develop an algorithm. In the smooth case a nonlinear evolution equation of fourth order arises because of the condition (1). Thus after partial integration in 14 we obtain

$$
X_{t}+\left(\Delta_{\Gamma}\right)^{2} X-2 \nabla_{\Gamma} \cdot\left(\Delta_{\Gamma} X \cdot v \nabla_{\Gamma} v\right)-\frac{1}{2} \nabla_{\Gamma} \cdot\left(\left|\Delta_{\Gamma} X\right|^{2} \nabla_{\Gamma} X\right)=0 .
$$


This is equivalent to

$$
X_{k t}+\left(\Delta_{\Gamma}\right)^{2} X_{k}-2 \nabla_{i}\left(\Delta_{\Gamma} X_{l} v_{l} \nabla_{i} v_{k}\right)-\frac{1}{2} \nabla_{i}\left(\left|\Delta_{\Gamma} X\right|^{2} \nabla_{i} X_{k}\right)=0
$$

for $k=1,2,3$.

An adequate solution space for the weak formulation would be the Sobolev space $H^{2}(S)^{3}$ and a finite element method would consist of $C^{1}$ elements. We wish to avoid this and to use a splitting into second order problems as developed in [8] for the fourth order Cahn-Hilliard equation and used in [7] for the elastic flow of curves. This will enable us to use piecewise linear finite elements.

But, to construct a discrete mixed method with Lagrange elements we observe that $\nabla_{\Gamma} v$ and therefore second order derivatives of $X$ are contained in the expression (14). These are not available for piecewise linear finite elements. Nevertheless the derivatives of $v$ can be eliminated by partial integration.

We introduce the new variable

$$
Y:=\Delta_{\Gamma} X
$$

which is the mean curvature vector, and rewrite the first variation. It is

$$
\int_{\Gamma} Y \cdot v \nabla_{\Gamma} v \cdot \nabla_{\Gamma} \varphi \mathrm{d} o=\int_{\Gamma} \nabla_{\Gamma} Y \cdot \nabla_{\Gamma} \varphi-\left(v \nabla_{\Gamma} Y\right) \cdot\left(v \nabla_{\Gamma} \varphi\right) \mathrm{d} o .
$$

To prove (17), we need basically to write the integrand on the left with the summation convention and apply the product rule. We use only geometrical quantities during this proof. A calculation gives

$$
\begin{aligned}
Y \cdot v \nabla_{\Gamma} v \cdot \nabla_{\Gamma} \varphi & =Y_{k} v_{k} \nabla_{i} v_{l} \nabla_{i} \varphi_{l} \\
& =\nabla_{i}\left(Y_{k} v_{k} v_{l} \nabla_{i} \varphi_{l}\right)-\nabla_{i} Y_{k} v_{k} v_{l} \nabla_{i} \varphi_{l}-Y_{k} \nabla_{i} v_{k} v_{l} \nabla_{i} \varphi_{l}-Y_{k} v_{k} v_{l} \nabla_{i} \nabla_{i} \varphi_{l} \\
& =\nabla_{\Gamma} \cdot\left(Y \nabla_{\Gamma} \varphi\right)-\left(\nu \nabla_{\Gamma} Y\right) \cdot\left(\nu \nabla_{\Gamma} \varphi\right)-Y \cdot \Delta_{\Gamma} \varphi
\end{aligned}
$$

pointwise in $\Gamma$. Since $Y$ is normal on $\Gamma$, we obtain (17) by using (12). We remark that the projection $\left(\delta_{i j}-v_{i} v_{j}\right)_{i, j=1,2,3}$ is included in (17) on the right. We insert this into 14p and get

Lemma 2.2 Let $X: \Omega \rightarrow \Gamma \subset \mathbb{R}^{3}$ be a parametrization of the regular $C^{3}$ surface $\Gamma$ such that $\Gamma=X(\Omega)$ and let $E$ be the energy functional defined in 13 . Then

$$
\begin{aligned}
\left\langle E^{\prime}(X), \varphi\right\rangle= & \left.\frac{\mathrm{d}}{\mathrm{d} \epsilon} E(X+\epsilon \varphi)\right|_{\epsilon=0} \\
= & \frac{1}{2} \int_{\Gamma}\left|\Delta_{\Gamma} X\right|^{2} \nabla_{\Gamma} X \cdot \nabla_{\Gamma} \varphi \mathrm{d} o \\
& +\int_{\Gamma} \nabla_{\Gamma}\left(\Delta_{\Gamma} X\right) \cdot \nabla_{\Gamma} \varphi-2\left(v \nabla_{\Gamma}\left(\Delta_{\Gamma} X\right)\right) \cdot\left(v \nabla_{\Gamma} \varphi\right) \mathrm{d} o
\end{aligned}
$$

for all $\varphi \in C_{0}^{2}(\Omega)^{3}$.

Proof. Apply Lemma 2.1 and 177 . 
Let us notice that now the reflection $\left(\delta_{i j}-2 v_{i} v_{j}\right)_{i, j=1,2,3}$ is contained in the first variation of $E$.

We formulate the continuous mixed problem by using Lemma 2.2. The calculations above can be applied to closed surfaces with arbitrary topological type by means of a partition of unity (see [17, p. 65]). Thus the mixed problem is posed for the position vector $X$ and the mean curvature vector $Y$ which are defined on a suitable surface $S$.

Problem 2.3 (Continuous mixed elastic flow) Let $X_{0}: S \rightarrow \Gamma_{0} \subset \mathbb{R}^{3}$ be a $C^{2}$ parametrization of the closed, regular, not necessarily embedded, $C^{2}$ surface $\Gamma_{0}=X_{0}(S)$ and let $Y_{0}:=\Delta_{\Gamma_{0}} X_{0}$ : $S \rightarrow \mathbb{R}^{3}$. Find $T>0$ and functions $X, Y: S \times[0, T] \rightarrow \mathbb{R}^{3}$ such that, at every time $t \in(0, T]$, $\Gamma=\Gamma(t)=X(S, t)$ is Lipschitz, $X(\cdot, t), Y(\cdot, t) \in H^{1}(S)^{3}, X_{t}(\cdot, t) \in L^{2}(S)^{3}$ with

$$
\int_{\Gamma} X_{t} \cdot \varphi \mathrm{d} o+\frac{1}{2} \int_{\Gamma}|Y|^{2} \nabla_{\Gamma} X \cdot \nabla_{\Gamma} \varphi \mathrm{d} o+\int_{\Gamma}\left(\nabla_{\Gamma} Y \cdot \nabla_{\Gamma} \varphi-2\left(v \nabla_{\Gamma} Y\right) \cdot\left(\nu \nabla_{\Gamma} \varphi\right)\right) \mathrm{d} o=0
$$

for all $\varphi \in H^{1}(\Gamma)^{3}$ and

$$
\int_{\Gamma} Y \cdot \psi \mathrm{d} o+\int_{\Gamma} \nabla_{\Gamma} X \cdot \nabla_{\Gamma} \psi \mathrm{d} o=0
$$

for all $\psi \in H^{1}(\Gamma)^{3}$ and $X(\cdot, 0)=X_{0}, Y(\cdot, 0)=Y_{0}$.

By comparing Problem 2.3 with the abstract initial value problem one sees that 19 is implied by the condition (1) for the velocity $v=X_{t}$ and 20 is the weak formulation of the definition of $Y$.

We next prove, under regularity assumptions, that the velocity $X_{t}$ in equation (19) is normal on $\Gamma$ at every time $t \in(0, T]$, i.e. $X_{t}$ has no tangential components. For that, we leave aside again the parametric representation of $\Gamma$ and make use only of geometrical quantities and of the relations between them.

We shift in (19) the derivatives of $\varphi$ to the other terms by 12] first and obtain an equality pointwise on $\Gamma$ which is equivalent to 16 . The definition of $Y$, the symmetry of the matrices $\nabla_{\Gamma} X=\left(\delta_{i j}-v_{i} v_{j}\right)_{i, j=1,2,3}$ and $\nabla_{\Gamma} v$, pointwise on $\Gamma$, and $9 p$ yield

$$
X_{t}=\left(\frac{1}{2} H^{3}-\Delta_{\Gamma} H-H\left|\nabla_{\Gamma} v\right|^{2}\right) v
$$

on $\Gamma$. It follows that

$$
X_{t}=-\left(\Delta_{\Gamma} H+\frac{1}{2} H^{3}-2 H K\right) v
$$

on $\Gamma$ by 10 . Consequently, we deduce, along the way, that the evolution law (2) for the normal velocity of the elastic flow for surfaces is fulfilled.

Lemma 2.2 remains valid if one looks at the elastic flow of curves. In this case the first variation (18) and with it the velocity condition (19) are equivalent to those presented in [7].

\section{Discrete formulation}

Through the calculation of $\left\langle E^{\prime}(X), \varphi\right\rangle$ a variational form for the continuous problem has been achieved. Thus finite element techniques can be applied. It is possible to use piecewise linear Lagrange elements since the mixed problem is formulated in $H^{1}(S)^{3}$.

The smooth surface $\Gamma$ will be approximated by a polyhedron $\Gamma_{h}=\bigcup_{T \subset \Gamma_{h}} T \subset \mathbb{R}^{3}$ of triangles $T \subset \Gamma_{h}$ such that $\bar{T}_{i} \cap \bar{T}_{j}=\partial T_{i} \cap \partial T_{j}$ for two different $T_{i}, T_{j} \subset \Gamma_{h}$ where $h=\max _{T \subset \Gamma_{h}} \operatorname{diam} T$ is 
the maximum mesh size. We require $\Gamma_{h}$ to be a closed, not necessarily embedded, Lipschitz surface. The Sobolev space $H^{1}\left(\Gamma_{h}\right)^{3}$ will be discretized by the finite element space

$$
\mathcal{X}_{h}\left(\Gamma_{h}\right):=\left\{\varphi \in C^{0}\left(\Gamma_{h}\right)^{3}: \varphi_{\mid T} \text { is a linear polynomial, } T \subset \Gamma_{h}\right\}
$$

and we have $\mathcal{X}_{h}\left(\Gamma_{h}\right) \subset H^{1}\left(\Gamma_{h}\right)^{3}$.

Problem 3.1 (Semi-discrete mixed elastic flow) Let $\Gamma_{h 0}$ be a closed initial $C^{0,1}$ polyhedron of triangles, $X_{h 0} \in \mathcal{X}_{h}\left(\Gamma_{h 0}\right)$ a parametrization of $\Gamma_{h 0}$, and $Y_{h 0} \in \mathcal{X}_{h}\left(\Gamma_{h 0}\right)$ with the property

$$
\int_{\Gamma_{h 0}} Y_{h 0} \cdot \psi \mathrm{d} o+\int_{\Gamma_{h 0}} \nabla_{\Gamma_{h 0}} X_{h 0} \cdot \nabla_{\Gamma_{h 0}} \psi \mathrm{d} o=0
$$

for all $\psi \in \mathcal{X}_{h}\left(\Gamma_{h 0}\right)$. Find, for $T>0$, closed $C^{0,1}$ polyhedrons $\Gamma_{h}(t)$ and functions $X_{h}(\cdot, t), X_{h t}(\cdot, t), Y_{h}(\cdot, t) \in \mathcal{X}_{h}\left(\Gamma_{h}(t)\right), 0 \leqslant t \leqslant T$, such that, for each $t \in(0, T], X_{h}$ is a parametrization of $\Gamma_{h}$ and

$\int_{\Gamma_{h}} X_{h t} \cdot \varphi \mathrm{d} o+\frac{1}{2} \int_{\Gamma_{h}}\left|Y_{h}\right|^{2} \nabla_{\Gamma_{h}} X_{h} \cdot \nabla_{\Gamma_{h}} \varphi \mathrm{d} o+\int_{\Gamma_{h}} \nabla_{\Gamma_{h}} Y_{h} \cdot \nabla_{\Gamma_{h}} \varphi-2\left(v_{h} \nabla_{\Gamma_{h}} Y_{h}\right) \cdot\left(v_{h} \nabla_{\Gamma_{h}} \varphi\right) \mathrm{d} o=0$

for all $\varphi \in \mathcal{X}_{h}\left(\Gamma_{h}\right)$

$$
\int_{\Gamma_{h}} Y_{h} \cdot \psi \mathrm{d} o+\int_{\Gamma_{h}} \nabla_{\Gamma_{h}} X_{h} \cdot \nabla_{\Gamma_{h}} \psi \mathrm{d} o=0
$$

for all $\psi \in \mathcal{X}_{h}\left(\Gamma_{h}\right)$, and $X_{h}(\cdot, 0)=X_{h 0}, Y_{h}(\cdot, 0)=Y_{h 0}$.

Let us remark that the discretization of $\Gamma_{h}(t)$ results from representing $\Gamma_{h}(t)$ by local parametrizations $X_{i}(t): \Omega_{i} \rightarrow \Gamma_{h}(t)$ where $\Omega_{i} \subset \mathbb{R}^{2}$ is a polygonal discrete parameter domain, $i=1, \ldots, i_{0}$, and $\bigcup_{i=1}^{i_{0}} X_{i}\left(\Omega_{i}, t\right)=\Gamma_{h}(t)$. One demands $\Omega_{i}$ to be time independent; for details see [12, p. 296].

Consider the time step $\tau>0$ with $T=m_{0} \tau$ for a $m_{0} \in \mathbb{N}$. We write $g^{m}:=g(\cdot, m \tau)$ for a function $g$ at time $m \tau, 0 \leqslant m \leqslant m_{0}$. The time discretization is now used to linearize the problem. Consequently, at each time step the integrals and the tangential gradients are built up with respect to the surface which is calculated a time step before, and a difference quotient is used instead the time derivative $X_{h t}$.

Problem 3.2 (Semi-implicit scheme for the elastic flow) Let $\Gamma_{h}^{0}$ be a closed Lipschitz polyhedron of triangles with parametrization $X_{h}^{0} \in \mathcal{X}_{h}\left(\Gamma_{h}^{0}\right)$ and let $Y_{h}^{0} \in \mathcal{X}_{h}\left(\Gamma_{h}^{0}\right)$ with

$$
\int_{\Gamma_{h}^{0}} Y_{h}^{0} \cdot \psi \mathrm{d} o+\int_{\Gamma_{h}^{0}} \nabla_{\Gamma_{h}^{0}} X_{h}^{0} \cdot \nabla_{\Gamma_{h}^{0}} \psi \mathrm{d} o=0
$$

for all $\psi \in \mathcal{X}_{h}\left(\Gamma_{h}^{0}\right)$. Find, for each $m=0,1, \ldots, m_{0}-1$, functions $X_{h}^{m+1}, Y_{h}^{m+1} \in \mathcal{X}_{h}\left(\Gamma_{h}^{m}\right)$ such that

$$
\begin{aligned}
\frac{1}{\tau} \int_{\Gamma_{h}^{m}}\left(X_{h}^{m+1}-X_{h}^{m}\right) \cdot \varphi \mathrm{d} o & +\frac{1}{2} \int_{\Gamma_{h}^{m}}\left|Y_{h}^{m}\right|^{2} \nabla_{\Gamma_{h}^{m}} X_{h}^{m+1} \cdot \nabla_{\Gamma_{h}^{m}} \varphi \mathrm{d} o \\
& +\int_{\Gamma_{h}^{m}} \nabla_{\Gamma_{h}^{m}} Y_{h}^{m+1} \cdot \nabla_{\Gamma_{h}^{m}} \varphi-2\left(v_{h}^{m} \nabla_{\Gamma_{h}^{m}} Y_{h}^{m+1}\right) \cdot\left(v_{h}^{m} \nabla_{\Gamma_{h}^{m}} \varphi\right) \mathrm{d} o=0
\end{aligned}
$$


for all $\varphi \in \mathcal{X}_{h}\left(\Gamma_{h}^{m}\right)$,

$$
\int_{\Gamma_{h}^{m}} Y_{h}^{m+1} \cdot \psi \mathrm{d} o+\int_{\Gamma_{h}^{m}} \nabla_{\Gamma_{h}^{m}} X_{h}^{m+1} \cdot \nabla_{\Gamma_{h}^{m}} \psi \mathrm{d} o=0
$$

for all $\psi \in \mathcal{X}_{h}\left(\Gamma_{h}^{m}\right)$ and

$$
\Gamma_{h}^{m+1}=X_{h}^{m+1}\left(\Gamma_{h}^{m}\right) .
$$

If we discretize $\Gamma_{h}^{m}$ as described in the semi-discrete case above, then $\Gamma_{h}^{m}$ can serve as reference surface for parametrizing $\Gamma_{h}^{m+1}$. This means we can define a global parametrization of $\Gamma_{h}^{m+1}$ over $\Gamma_{h}^{m}$. Obviously, it suffices to parametrize locally only $\Gamma_{h}^{0}$; for details see [6].

Accordingly, at each time step a linear equation system has to be solved. The system is coupled mainly because of the reflection. The matrices are symmetric.

\section{Conclusion}

Our aim was to derive a mixed variational problem for the elastic flow of a closed two-dimensional surface $\Gamma$ in $\mathbb{R}^{3}$ which is suitable for discretization with linear finite elements. We wanted to obtain a scheme analogous to the semi-implicit fully discrete finite element scheme for the elastic flow of curves developed in [7]. We succeeded by calculating the gradient of the elastic energy $E$ (see Lemmas 2.1 and 2.2. A semi-discretization and a full discretization are formulated in Problems 3.1 and 3.2 .

\section{Acknowledgements}

I thank my supervisor Prof. Dr. G. Dziuk for interesting discussions and support.

\section{REFERENCES}

1. Aubin, T. Nonlinear Analysis on Manifolds. Monge-Ampère Equations. Springer, New York (1982). Zbl 0512.53044 MR 0681859

2. Do CARmo, M. P. Differentialgeometrie von Kurven und Flächen. Vieweg, Braunschweig/Wiesbaden (1983). Zbl 0606.53002 MR 0726220

3. Clarenz, U., Diewald, U., Dziuk, G., Rumpf, M. \& Rusu, R. A finite element method for surface restoration with smooth boundary conditions. Comput. Aided Geom. Design 21 (2004), 427-445. MR 2058390

4. Deckelnick, K. \& DZiuk, G. Error estimates for the Willmore flow of graphs. Preprint, Fakultät für Mathematik und Physik, Universität Freiburg, Nr. 25-04 (2004).

5. DZIUK, G. Finite elements for the Beltrami operator on arbitrary surfaces. Partial Differential Equations and Calculus of Variations, S. Hildebrandt and R. Leis (eds.), Lecture Notes in Math. 1357, Springer, Berlin (1988), 142-155. Zbl 0663.65114 MR 0976234

6. DZIUK, G. An algorithm for evolutionary surfaces. Numer. Math. 58 (1991), 603-611. Zbl 0714.65092 MR 1083523

7. Dziuk, G., Kuwert, E., \& Schätzle, R. Evolution of elastic curves in $\mathbb{R}^{n}$ : Existence and computation. SIAM J. Math. Anal. 33 (2002), 1228-1245. Zbl 1031.53092 MR 1897710

8. Elliott, C. M., French, D., \& Milner, F. A second order splitting method for the Cahn-Hilliard equation. Numer. Math. 54 (1989), 575-590. Zbl 0668.65097 MR 0978609 
9. Gilbarg, D. \& Trudinger, N. S. Elliptic Partial Differential Equations of Second Order. Springer, Berlin (1983). Zbl 0562.35001 MR 0737190

10. Kuwert, E. \& Schätzle, R. Gradient flow for the Willmore functional. Comm. Anal. Geom. 10 (2002), 307-339. Zbl 1029.53082 MR 1900754

11. Polden, A. Curves and surfaces of least total curvature and fourth-order flows. Dissertation, Mathematisches Institut, Universität Tübingen (1996).

12. Schmidt, A. Computation of three dimensional dendrites with finite elements. J. Comput. Phys. 125 (1996), 293-312. Zbl 0844.65096

13. Simonett, G. \& MAYER, U. F. A numerical scheme for radially symmetric solutions of curvature driven free boundary problems, with applications to the Willmore flow. Interfaces Free Bound. 4 (2002), 89-109. Zbl 1005.65095 MR 1877537

14. Willmore, T. J. Note on embedded surfaces. An. Şti. Univ. “Al. I. Cuza” Iaşi Secţ. Ia Mat. (N.S.) 11B (1965), 493-496. Zbl 0171.20001 MR 0202066

15. Willmore, T. J. Mean curvature of immersed surfaces. An. Şti. Univ. “Al. I. Cuza” Iaşi Secţ. Ia Mat. (N.S.) 14 (1968), 99-103. Zbl 0163.43002 MR 0238220

16. Willmore, T. J. Riemannian Geometry. Clarendon Press, Oxford (1993). Zbl 0797.53002 MR 1261641

17. Wloka, J. Partielle Differentialgleichungen. B. G. Teubner, Stuttgart (1982). Zbl 0482.35001 MR 0652934 